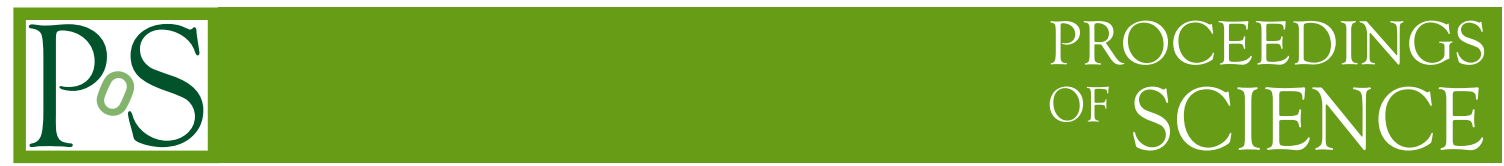

\title{
ATLAS studies of spectroscopy and exotics
}

\section{Semen Turchikhin, ${ }^{a, *}$ on behalf of the ATLAS collaboration}

${ }^{a}$ Joint Institute for Nuclear Research, Dubna, Russia

E-mail: Semen.Turchikhin@cern.ch

This proceedings contribution is devoted to studies of heavy flavour hadrons spectroscopy and exotic hadron states in the ATLAS experiment at the LHC. It presents in details the result of a recent study of resonances in $J / \psi p$ system using $p p$ collision data collected during LHC Run-1 at $\sqrt{s}=7$ and $8 \mathrm{TeV}$ performed to test the existence of the pentaquark states observed earlier by the $\mathrm{LHCb}$ experiment.

40th International Conference on High Energy physics - ICHEP2020

July 28 - August 6, 2020

Prague, Czech Republic (virtual meeting)

\footnotetext{
${ }^{*}$ Speaker
} 


\section{Introduction}

The programme of the ATLAS experiment [1] at the LHC in heavy flavour physics is mainly focused on studying final states with two muons, due to the availability of corresponding triggers with relatively low muon thresholds. Although limiting the scope of possible physics analyses, this still leaves a lot of opportunities, in particular for hadron spectroscopy measurements and exotic hadrons studies, as they often involve heavy quarkonium states decaying to a muon pair. A number of results has been published by ATLAS since the start of LHC operation, including first observation of $B_{c}(2 S)$ excited state [2] or measurement of $X(3872)$ production [3].

Pentaquarks, baryons composed of four quarks and one antiquark, have attracted a particular attention in 2015 when LHCb has observed two resonant structures in $J / \psi p$ system produced in the $\Lambda_{b}^{0} \rightarrow J / \psi p K^{-}$decay interpreted as $c \bar{c} u u d$ pentaquark states $P_{c}(4380)^{+}$and $P_{c}(4450)^{+}$[4]. Their existence has been later confirmed in a model-independent analysis [5]. After studying a larger dataset $\mathrm{LHCb}$ has recently reported that the $P_{c}(4450)^{+}$signal may represent two narrower states, $P_{c}(4440)^{+}$and $P_{c}(4457)^{+}$, and that there is another narrow resonance, $P_{c}(4312)^{+}$[6]. These narrow states have masses close to the $\bar{D}^{(*)} \Sigma_{c}^{(*)}$ mass threshold that is in agreement with the prediction of dynamically generated meson-baryon narrow states with hidden charm [7], while status and nature of the wider $P_{c}(4380)^{+}$state is less clear.

This report presents a study of the $J / \psi p$ resonances in the $\Lambda_{b}^{0} \rightarrow J / \psi p K^{-}$decays by the ATLAS experiment [8]. The analysis is based on a combined sample of $p p$ collision data at centre-of-mass energies $\sqrt{s}=7 \mathrm{TeV}$ and $8 \mathrm{TeV}$ collected during LHC Run-1.

\section{2. $J / \psi p$ resonances analysis strategy}

Due to ATLAS inability to distinguish the tracks produced by different charged hadrons, the $\Lambda_{b}^{0}$ decays are reconstructed together with other $b$ hadrons $\left(H_{b}\right)$ decays: $B^{0} \rightarrow J / \psi K^{+} \pi^{-}$, $B^{0} \rightarrow J / \psi \pi^{+} \pi^{-}, B_{s}^{0} \rightarrow J / \psi K^{+} K^{-}$, and $B_{s}^{0} \rightarrow J / \psi \pi^{+} \pi^{-}$. The invariant mass of the two hadronic tracks with $\pi K$ hypothesis is required to be $m(\pi K)>1.55 \mathrm{GeV}$, corresponding to $m(p K)$ approximately above $2 \mathrm{GeV}$. This allows to substantially suppress a large background from contribution of intermediate light $\Lambda^{*}, K^{*}$ and other states in the $H_{b}$ decays.

Monte Carlo simulation of all mentioned $H_{b}$ decays is used for subsequent fits to data. The simulation is done using so-called "phase-space" model for the decays, and the events are weighted according to analytically derived matrix elements for those decays. The matrix element is a coherent sum of helicity amplitudes of all resonant contributions to the corresponding $H_{b}$ decay, including all known resonances $\left(\Lambda^{*}, K^{*}, f\right.$, and $\phi$ states) and, optionally, new $P_{c}$ states in the $\Lambda_{b}^{0}$ decay. Interference and spin effects in the decay chains are properly taken into account. A set of decay coupling constants as well as masses and widths of the new states in the matrix element are free parameters and defined from the fits to data described below.

One signal and two control regions are used for the data fits. The signal region is defined in terms of $J / \psi p K$ invariant mass to be enriched with $\Lambda_{b}^{0} \rightarrow J / \psi p K^{-}$decay events. Two control regions enriched with the $B^{0}$ and $B_{s}^{0}$ decay events are defined in terms of $m(J / \psi \pi K)$ and $m(J / \psi K K)$ masses, respectively. 
Due to the complexity of the decay model, the data fit is done iteratively. Each iteration includes four steps. The parameter values obtained in each step are used in the following step. After the fourth step, the new iteration starts with step one. The steps are:

1. "Global" fit of two-dimensional distributions of masses of the $J / \psi$ with one or both hadrons in the full selected dataset, and the two hadron tracks mass distributions in the $B^{0}$ and $B_{s}^{0}$ control regions, assuming various kaon/pion mass hypotheses. The minimized funcion is a sum of the binned log-likelihoods for each of the involved distributions. At this step the coupling constants of the $B^{0}$ and $B_{s}^{0}$ decays are extracted, with the $\Lambda_{b}^{0}$ decay parameters fixed to the values from the previous iterations.

2. The chi-square fit of the $m(J / \psi p K)$ mass distribution to extract the yields of $\Lambda_{b}^{0}, B^{0}$, and $B_{s}^{0}$ decays and of the combinatorial background. All decay couplings are fixed at this step.

3. Fit of the two-dimensional distribution of $m(J / \psi p)$ versus $m(J / \psi K)$ and of $m(p K)$ in the signal region, minimizing the sum of binned log-likelihoods for both. The purpose of this step is to extract the decay constants for the $\Lambda_{b}^{0}$ decays.

4. The chi-square fit of the $m(J / \psi p)$ distribution in the signal region to extract the yields, masses and width of the $P_{c}$ states.

To suppress the effect of combinatorial background, all fits are performed after subtracting the pure background contribution from combinations with same charge of the two hadron tracks from the contribution of the combinations with the opposite hadron charges.

Figure 1 (left) shows the $m(J / \psi p K)$ distribution for all selected $\Lambda_{b}^{0}$ candidates used at step 2 of the iterative procedure and the results of the fit. The total $\Lambda_{b}^{0}$ yield is $2270 \pm 300$, while $1010 \pm 140$ $(160 \pm 20)$ signal decays with right (wrong) proton/kaon mass assignment fall inside the signal region.
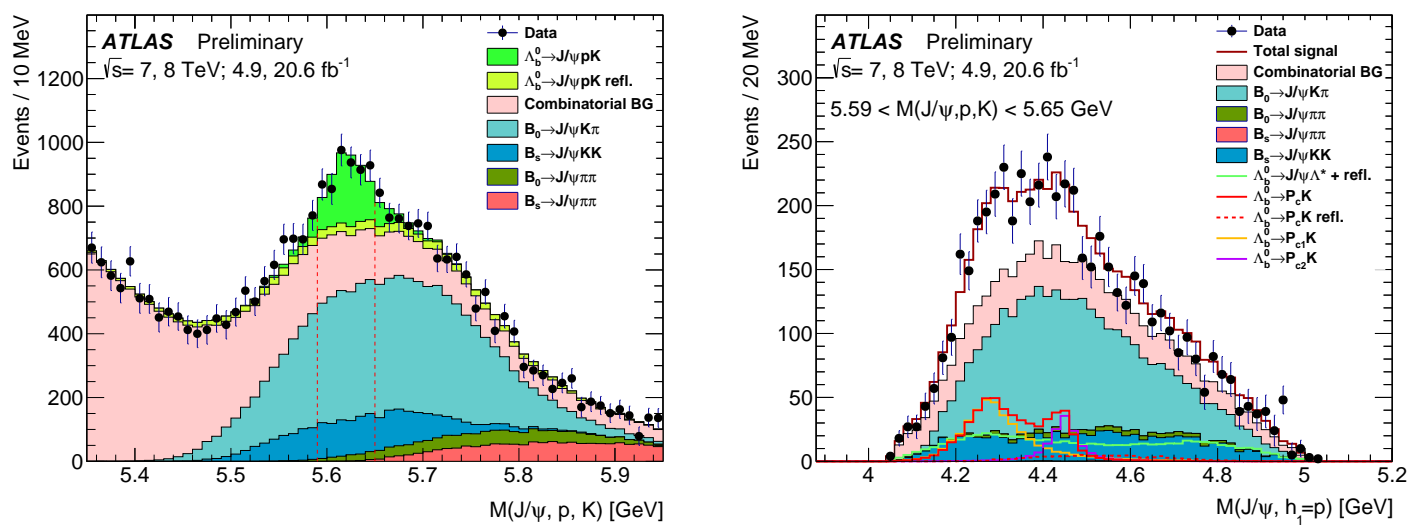

Figure 1: Left: The invariant mass $m(J / \psi p K)$ distribution for all selected $\Lambda_{b}^{0}$ candidates with the result of the iterative fit procedure overlaid; red dashed lines indicate the signal region boundaries. Right: Result of the chi-square fit of the $m(J / \psi p)$ distribution in the signal region with the hypothesis of two pentaquarks $P_{c 1}$ and $P_{c 2}$ with $J^{P}=3 / 2^{-}$and $5 / 2^{+}$, respectively. Figures taken from Ref. [8]. 


\section{Results}

Parameters of the $P_{c}$ states are extracted using the nominal hypothesis of the existance of the two pentaquarks with $J^{P}=3 / 2^{-}$and $5 / 2^{+}$for the lighter and heavier state, respectively, as reported in Ref. [4]. Figure 1 (right) shows the result of the $m(J / \psi p)$ distribution fit under this hypothesis. The data description is good $\left(\chi^{2} /\right.$ n.d.f. $\left.=37.1 / 39\right)$ corresponding to a $p$-value of $55.7 \%$.

Table 1: Parameters of the $\Lambda_{b}^{0} \rightarrow P_{c}^{+} K^{-}$decays derived from the data fit for the hypothesis with two pentaquarks, in comparison with the values reported by LHCb [4]. Table taken from Ref. [8].

\begin{tabular}{lcc}
\hline Parameter & \multicolumn{1}{c}{ Value } & LHCb value \\
\hline$N\left(P_{c 1}\right)$ & $400_{-140}^{+130}$ (stat. $)_{-100}^{+110}$ (syst.) & - \\
$N\left(P_{c 2}\right)$ & $150_{-100}^{+170}$ (stat. $)_{-90}^{+50}$ (syst.) & - \\
$N\left(P_{c 1}+P_{c 2}\right)$ & $540_{-70}^{+80}$ (stat. $)_{-80}^{+70}$ (syst.) & - \\
$\Delta \phi$ & $2.8_{-1.6}^{+1.0}$ (stat. $)_{-0.1}^{+0.2}$ (syst.) rad & - \\
$m\left(P_{c 1}\right)$ & $4282_{-26}^{+33}$ (stat. $)_{-7}^{+28}$ (syst.) $\mathrm{MeV}$ & $4380 \pm 8 \pm 29 \mathrm{MeV}$ \\
$\Gamma\left(P_{c 1}\right)$ & $140_{-50}^{+77}$ (stat. $)_{-33}^{+41}$ (syst.) $\mathrm{MeV}$ & $205 \pm 18 \pm 86 \mathrm{MeV}$ \\
$m\left(P_{c 2}\right)$ & $4449_{-29}^{+20}$ (stat. $)_{-10}^{+18}$ (syst.) $\mathrm{MeV}$ & $4449.8 \pm 1.7 \pm 2.5 \mathrm{MeV}$ \\
$\Gamma\left(P_{c 2}\right)$ & $51_{-48}^{+59}$ (stat. $)_{-46}^{+14}$ (syst.) $\mathrm{MeV}$ & $39 \pm 5 \pm 19 \mathrm{MeV}$ \\
\hline
\end{tabular}

The obtained yields of the pentaquark states, their masses, widths, and the relative phase are shown in Table 1. The masses and widths are consistent with those obtained by LHCb within uncertainties. The data fit with these parameters fixed to the LHCb values yields $\chi^{2} /$ n.d.f. $=49.0 / 43$ corresponding to a $p$-value of $24.5 \%$.

The narrow pentaquarks reported by LHCb later [6] cannot be distinguished in the ATLAS analysis due to the smaller number of signal candidates and the $m(J / \psi p)$ resolution achieved. To verify whether the data are compatible with the new LHCb observations, the fit is performed with a four pentaquarks hypothesis, while masses, widths and relative yields of the narrow pentaquarks are fixed to the LHCb values [6]. Result of such fit is shown in Figure 2 (left); the data description with this hypothesis is equally good $\left(\chi^{2} /\right.$ n.d.f. $\left.=37.1 / 39\right)$.

The fit is repeated using the $\Lambda_{b}^{0}$ decay model without pentaquarks. This yields $\chi^{2} /$ n.d.f. $=$ $69.2 / 37$ corresponding to a $p$-value of $1.0 \times 10^{-3}$. The null hypothesis fit is performed for a set of systematic variations of the nominal fit model as well. This best data description is achieved with an extended $\Lambda_{b}^{0} \rightarrow J / \psi \Lambda^{* 0}$ decay model which allows two lowest orbital momenta between the decay products of certain $\Lambda^{*}$ resonances (only the lowest orbital momentum is allowed in the nominal model). The $\chi^{2}$ fit of the $m(J / \psi p)$ distribution with this alternative model shown in Figure 2 (right) yields $\chi^{2} /$ n.d.f. $=42.0 / 23$ and a $p$-value of $9.1 \times 10^{-3}$. The description of data is not as good as for the hypothesis with two pentaquarks, though acceptable. 

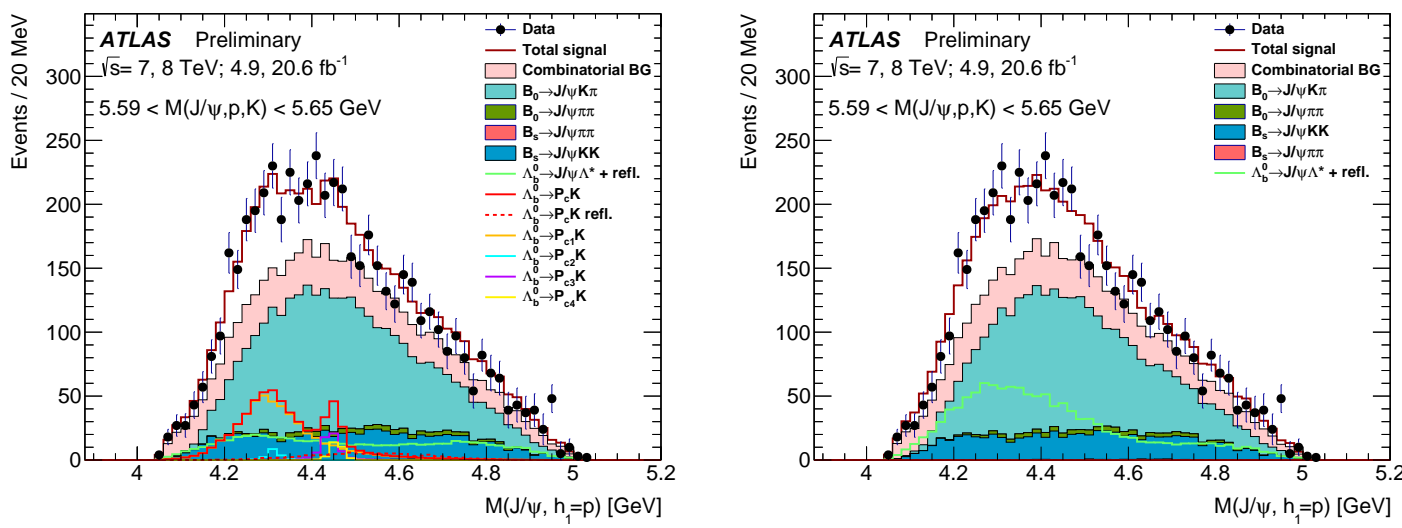

Figure 2: Left: Result of the $\chi^{2}$ fit of the $m(J / \psi p)$ distribution in the signal region with the hypothesis of four pentaquarks $P_{c 1}, P_{c 2}, P_{c 3}, P_{c 4}$. Right: Result of the same fit for the model without pentaquarks and with extended $\Lambda_{b}^{0} \rightarrow J / \psi \Lambda^{*}$ decays description. Figures taken from Ref. [8].

\section{Summary}

The ATLAS study of $J / \psi p$ resonances in the $\Lambda_{b}^{0} \rightarrow J / \psi p K^{-}$decays with Run-1 data has been presented in this report. Using the data fit model with two pentaquark states, their masses and widths are found compatible with the corresponding result of the LHCb experiment [6]. The data are also consistent with the more recent LHCb observation of three narrow pentaquark states [6], although an independent measurement of their parameters is not possible yet. Although the data prefer the model with two or more pentaquarks, the model without pentaquarks cannot be excluded by the performed analysis.

Sensitivity to the pentaquark states should be improved with the dataset collected during LHC Run-2 where ATLAS can benefit from much larger statistics as well as from detector and trigger improvements. The Run-2 dataset provides new opportunities for many other hadron spectroscopy studies, related both to exotics and conventional hadrons such as $B_{c}$ meson excited states.

\section{Acknowledgements}

The work presented was partially supported by RFBR according to the research project 18-3200825 .

\section{References}

[1] ATLAS Collaboration, The ATLAS Experiment at the CERN Large Hadron Collider, JINST 3 (2008) S08003.

[2] ATLAS Collaboration, Observation of an Excited $B_{c}^{ \pm}$Meson State with the ATLAS Detector, Phys. Rev. Lett. 113 (2014) 212004, arXiv: 1407.1032 [hep-ex]. 
[3] ATLAS Collaboration, Measurements of $\psi(2 S)$ and $X(3872) \rightarrow J / \psi \pi^{+} \pi^{-}$production in $p p$ collisions at $\sqrt{s}=8 \mathrm{TeV}$ with the ATLAS detector, JHEP 01 (2017) 117, arXiv: 1610.09303 [hep-ex].

[4] LHCb Collaboration, Observation of $J / \psi$ p Resonances Consistent with Pentaquark States in $\Lambda_{b}^{0} \rightarrow J / \psi K^{-} p$ Decays, Phys. Rev. Lett. 115 (2015) 072001, arXiv: 1507.03414 [hep-ex].

[5] LHCb Collaboration, Model-independent evidence for $J / \psi p$ contributions to $\Lambda_{b}^{0} \rightarrow J / \psi p K^{-}$ decays, Phys. Rev. Lett. 117 (2016) 082002, arXiv: 1604.05708 [hep-ex].

[6] LHCb Collaboration, Observation of a narrow pentaquark state, $P_{c}(4312)^{+}$, and of two-peak structure of the $P_{c}(4450)^{+}$, Phys. Rev. Lett. 122 (2019) 222001, arXiv: 1904.03947 [hep-ex].

[7] J.-J. Wu, R. Molina, E. Oset, and B. Zou, Prediction of narrow $N^{*}$ and $\Lambda^{*}$ resonances with hidden charm above 4 GeV, Phys. Rev. Lett. 105 (2010) 232001, arXiv: 1007.0573 [nucl-th].

[8] ATLAS Collaboration, Study of $J / \psi$ p resonances in the $\Lambda_{b}^{0} \rightarrow J / \psi p K^{-}$decays in $p p$ collisions at $\sqrt{s}=7$ and $8 \mathrm{TeV}$ with the ATLAS detector, ATLAS-CONF-2019-048, https://cds. cern.ch/record/2693957. 